\title{
Hidropolítica en las zonas áridas y semiáridas de los Andes de América del Sur
}

\author{
Manuel de Cossío Klüver **
}

\section{RESUMEN}

De acuerdo con el conocimiento convencional es probable que la competencia interestatal por acceder a las escasas fuentes de agua dulce planetarias se convierta en una de las principales preocupaciones de los gobiernos y la población mundial en las próximas décadas. De cumplirse esa predicción, se podrían incrementar las tensiones ya existentes entre los Estados cuando se ańada una competencia más intensa por los recursos hídricos ubicados en sus fronteras. Estas tensiones se harían más agudas en zonas áridas o semiáridas, como los Andes y la costa del Pacífico Sur, en donde existe una escasez crónica de agua dulce al igual que estrés hídrico, donde el retroceso de los glaciares y las presiones que se ejercen sobre los limitados recursos hídricos existentes magnifican el panorama de escasez general y, por ende, la tensión hídrica. Este artículo es parte de un estudio sobre las tensiones hídricas emergentes del uso y gestión de recursos hídricos transfronterizos en zonas áridas y semiáridas, así como sobre su impacto en la seguridad de los Estados que comparten cursos de agua internacionales. En este texto se utilizará el caso del sistema hidrográfico del Silala, ubicado en la puna del desierto de Atacama, con la finalidad de analizar los factores políticos que podrían desencadenar conflictos hídricos, así como dificultar la adopción de acuerdos cooperativos.

Palabras clave: acuíferos, agua, Andes, Bolivia, Chile, CIJ, conflicto, cooperación, hidropolítica, Silala.

\footnotetext{
Magíster en Derecho Internacional Público, Relaciones Internacionales y Diplomacia. Actualmente es candidato a Doctor en Derecho en la Universidad de Montreal, Quebec, Canadá. Ministro en el Servicio Diplomático del Perú. Correo electrónico: mdecossio@videotron.ca

(iD) https://orcid.org/g/0000-0003-4077-2147

** Las opiniones expresadas en este artículo representan únicamente los puntos de vista personales del autor y no representan los puntos de vista de las instituciones con las que el autor está asociado.
} 
Hydro politics in the arid and semi-arid zones of South American Andes

\section{Abstract}

According to conventional knowledge, interstate competition to access scarce planetary freshwater sources is likely to become one of the main concerns of governments and the world's population in the coming decades. If this prediction is fulfilled, the already existing tensions between States could increase when more intense competition is added for the water resources located on their borders. These tensions would become more acute in arid or semi-arid areas, such as the Andes and the South Pacific coast, where there is a chronic shortage of fresh water as well as water stress, where the retreat of glaciers and the pressures that are exerted on limited existing water resources magnify the overall scarcity picture, and thus water stress. This article is part of a study on the emerging water stresses of the use and management of transboundary water resources in arid and semi-arid zones, as well as its impact on the security of the States that share international watercourses. The case of the Silala hydrographic system, located in the puna of the Atacama Desert, will be used to analyze the political factors that could trigger water conflicts, as well as hinder the adoption of cooperative agreements.

Keywords: Aquifers, Andes, Bolivia, Chile, cooperation, conflict, hydro politics, ICJ, Silala, water.

\section{Introducción}

Es probable que la competencia por la utilización de recursos hídricos limitados se convierta en las siguientes décadas en una de las grandes preocupaciones que existan en las relaciones internacionales (Gallopín, 2012). Si bien es cierto que las tensiones interestatales alrededor del agua no han sido la causa principal de conflictos armados en el pasado (Dolatyar, 1 de agosto de 2002; Otchet, 2001, pp. 18-19; Gleick, 16 de agosto de 2020) no se puede descartar que en el futuro - a medida que la población mundial se incremente, se multipliquen las demandas por el uso y gestión del agua dulce y se hagan más evidentes los efectos del cambio climático- estas tensiones se conviertan en uno de los factores que expliquen buena parte de los conflictos que podrían surgir entre los Estados ribereños de un curso de agua (Gleick e Iceland, 2018; UN-WATER, 2013; Ganter, 2015; Gleick, Iceland y Trivendi, 2020; Intelligence Community Assessment [ICA], 2012; World Economic Forum [WEF], 2018).

En ese contexto, las tensiones hídricas interestatales pueden exacerbar y complicar aún más las tensiones políticas, económicas y sociales ya existentes; incrementando de esa manera la inestabilidad y el malestar político en varias regiones del planeta, sobre todo en aquellas que ya sufren estrés hídrico o escasez crónica de agua como las zonas áridas y semiáridas del planeta. 
Esas regiones son vulnerables y propensas a acumular tensiones hídricas relativas al manejo, gestión y uso del agua dulce. La literatura académica sobre hidropolítica en los cursos de agua internacionales identifica a varios ríos ${ }^{1}$ y acuíferos ${ }^{2}$ como los ejemplos más relevantes de conflictos políticos que existen en la actualidad en cursos de agua ubicados en este tipo de zonas (Wong et al., 2007; Gleick e Iceland, 2018; Reed, 2017; UN-WATER, 2013; ICA, 2012; Gleick, Iceland y Trivendi, 2020; WEF, 2018).

A pesar de la abundancia de estudios sobre hidropolítica, son muy pocos los que prestan atención a los conflictos existentes en los ríos y acuíferos ubicados en las zonas áridas y semiáridas de América del Sur, en particular aquellos ubicados en la costa desértica del Perú o en el desierto de Atacama en Chile. El que ha tenido mayor influencia es el realizado por Newton (2007) sobre la vulnerabilidad y resiliencia hidropolíticas en América Latina y el Caribe. Ese estudio utiliza los parámetros teóricos escogidos por Wolf (2007) para medir la resiliencia y la vulnerabilidad hidropolítica en cuencas transfronterizas sudamericanas.

Aplicando esos conceptos a los principales cursos de agua sudamericanos (como el Amazonas, La Plata, el Orinoco, el lago Titicaca y el acuífero Guaraní) Wolf y Newton evalúan su grado de vulnerabilidad y resiliencia hidropolítica. Concluyen que esas cuencas contienen una gran proporción de los recursos hídricos del subcontinente y presentan una buena resiliencia hidropolítica. Asimismo, señalan que cuencas pequeñas y con escaso caudal — como las del Lauca y del Silala— «son dos ejemplos de aguas transfronterizas que han visto mucho conflicto, y es posible que vean más en el futuro debido a la región seca en la que se ubican» (Newton, 2007, p. 50). Ambos cursos de agua están situados en la puna colindante con el desierto de Atacama en la frontera boliviano-chilena.

Newton sostiene que el sistema hidrográfico del Silala es un ejemplo de la manera en que «las fuerzas políticas y socioeconómicas pueden causar una disputa sobre un cuerpo de agua» (Newton, 2007, p. 64). Añade que, si bien este conflicto entre Bolivia y Chile se refiere al uso del agua del Silala, son los "problemas subyacentes» los que lo han convertido en una de las cuencas hidropolíticamente más vulnerables en el mundo ${ }^{3}$.

\footnotetext{
1 Como el Nilo, el Tigris, el Éufrates, el Murray/Darling, el Indo, el Amu-Darya, el Sir- Darya, el Grande/Bravo, el Colorado, el Yarmuk y el Jordán, entre otros.

2 Como el Ertyx (China/Kazajstán), el Irtyst-Obsky (Rusia/Kazajstán), el del sistema del Noroeste del Sahara (Libia/Argelia), el del sistema Arenisco Nubio (Chad/Sudán/Egipto/Libia), el sistema Umm er Radhuna-Damman (Omán/Arabia Saudita/Emiratos Árabes Unidos/Yemen), entre otros.

3 Por "problemas subyacentes», Newton (2007) se refiere a factores políticos, económicos, demográficos e históricos, así como a conflictos de soberanía que trascienden la gobernanza del agua.
} 
Cabe mencionar que la caracterización de vulnerabilidad hidropolítica que Newton atribuye al Silala ha sido utilizada, por ejemplo, por investigadores asociados a la Academia Nacional de Estudios Políticos y Estratégicos (ANEPE) del Ministerio de Defensa Nacional de Chile para explicar el alto grado de conflictividad existente con Bolivia en torno a la utilización del agua del Silala (Faundes Sánchez, 2008; Correa Vera y Salas Maturana, 2015; Correa Vera, 2019; Zambrano, 2015).

En las siguientes secciones profundizaremos en los argumentos a favor de atribuir un alto riesgo hidropolítico a un sistema hidrográfico muy pequeño, de escaso caudal, ubicado en las cumbres andinas del desierto de Atacama sobre los 3800 metros s. n. m., en el que no se han desarrollado proyectos hidráulicos mayores desde 1908, que no cuenta con un marco institucional bilateral y que provee de agua a través de un sistema de canales a varias poblaciones del norte chileno y, principalmente, al complejo minero de Chuquicamata ${ }^{4}$ el yacimiento cuprífero más importante de la Corporación Nacional del Cobre de Chile (CODELCO, 2019). Para este fin utilizaremos como referente teórico la literatura académica sobre hidropolítica.

\section{Hidropolítica: más allá del conflicto y la cooperación en torno a los cursos de agua internacionales}

Una visión corriente y muy difundida sobre el conflicto y la cooperación por el agua dulce la da, por ejemplo, el proyecto de la Unesco denominado «Del Conflicto Potencial a la Cooperación Potencial» (PCCP por sus siglas en inglés). Este proyecto de las Naciones Unidas señaló que aun cuando exista una muy alta posibilidad que se produzcan conflictos en torno al uso de los cursos de agua internacionales es también posible utilizar la gestión conjunta de sus recursos hídricos para fortalecer la cooperación entre los Estados y superar los conflictos hidropolíticos que se producen en cuencas transfronterizas 5 .

Desde esta perspectiva, la cooperación interestatal se transforma en una herramienta fundamental para facilitar que los conflictos por el agua se solucionen teniendo en cuenta los intereses de todas las partes interesadas (stakeholders). En esta línea de pensamiento, prevenir y evitar los conflictos que puedan surgir entre los Estados como resultado de sus demandas por el acceso al agua y la posible utilización desigual

\footnotetext{
4 Integrado por las explotaciones mineras Chuquicamata, Radomiro Tomic y Ministro Hales ubicadas a 99 kilómetros en línea recta de los manantiales del Silala y cercanas a la ciudad chilena de Calama. Estas tres minas producen más de 900 mil toneladas métricas de cobre anualmente.

5 Unesco. «PCCP. From Potential Conflict to Cooperartion Potential», Institutional, International Hydrological Programme, accedido 27 de septiembre de 2018, http://www.unesco.org/new/en/natural-sciences/environment/ water/ihp/ihp-programmes/pccp/.
} 
de los recursos hídricos para una variedad de usos es una tarea imprescindible. Este punto de vista parece ubicar en una secuencia lineal el tránsito desde una situación de conflicto hacia una de cooperación por los recursos hídricos transfronterizos.

La literatura académica sobre el conflicto y la cooperación interestatal en torno a los cursos de agua internacionales se asocia frecuentemente con estudios sobre: a) agua y conflicto, b) agua y medio ambiente, c) agua y seguridad y d) agua, sociedad y cultura. Cada uno de estos enfoques teóricos presenta, de acuerdo con Turton y Henwood, perspectivas teóricas específicas y distintivas (Turton y Henwood, 2002).

Para los efectos de este artículo utilizaremos la perspectiva teórica de los estudios sobre agua y conflicto, en particular aquellos centrados en el estudio de la hidropolítica. Waterbury (1979) fue uno de los primeros en utilizar el concepto de hidropolítica aplicándolo al caso del río Nilo. Este académico lo concibió como la capacidad asociada a las instituciones con carácter geopolítico (es decir los Estados) para manejar y gestionar los recursos hídricos transfronterizos de una manera sostenible, es decir, administrarlos de una forma que todos los Estados involucrados reciban el recurso, disminuya su despilfarro y se eviten conflictos relacionados a su acceso (Waterbury, 1979).

Se debe notar, sin embargo, que existen otras definiciones de hidropolítica que han ido ampliando la pionera definición elaborada por Waterbury. Por ejemplo, una de ellas es la proporcionada por Elhance (1999), quien señaló que se trata del análisis sistemático del conflicto y la cooperación interestatales respecto a los recursos hídricos transfronterizos. Otra es la diseñada por Meissner (2016), quien nos ofrece una definición más elaborada de hidropolítica. Este autor la percibe como la investigación sistemática de la interacción entre Estados, actores no estatales y una gran cantidad de otros participantes (partes interesadas / stakeholders) directamente vinculados a la asignación autorizada y/o uso de recursos hídricos nacionales y transfronterizos.

En resumen, tal como se conceptúa actualmente, la hidropolítica tiende a enfocarse en los conflictos que pueden surgir en cursos de agua internacionales, principalmente a nivel interestatal y entre actores de diverso tipo, así como en las vías de cooperación para solucionarlos (Turton y Henwood, 2002).

\section{Indicadores para medir la dicotomía conflicto/cooperación en cursos de agua internacionales: factores que cuantifican la tensión hidropolítica}

A partir de la última década del siglo XX se han desarrollado diferentes metodologías para intentar medir la tensión hidropolítica e identificar, a nivel mundial, las zonas de potencial conflicto/cooperación entre los Estados ribereños de un curso de agua 
internacional (UNEP, 2007; ICA, 2012; Gleick e Iceland, 2018; Gleick, Iceland, y Trivedi, 2020). Estas metodologías utilizan bases de datos en las que se registra información sobre los indicadores que identificarían las interacciones hidropolíticas en cursos de agua internacionales ${ }^{6}$. Los indicadores que han sido utilizados para medir la tensión e interacción hidropolítica son variados, no siempre coincidentes y, por lo general, no hacen referencia a «los problemas subyacentes» identificados por Newton.

Recientemente se han realizado estudios cuantitativos más consistentes para intentar medir cuáles podrían ser los factores más relevantes para determinar la existencia de interacciones hidropolíticas en cursos de agua internacionales (De Stefano et al., 2017; Farinosi et al., 2018). Estos análisis utilizan varios indicadores para medir la existencia y cualidad de las interacciones hidropolíticas definidas como todos los episodios pasados de conflicto y cooperación sobre recursos hídricos transfronterizos (Farinosi et al., 2018).

Se debe mencionar que, si bien estos estudios identifican con claridad algunos indicadores que, junto con otros, explicarían la actual tensión hidropolítica existente en el Silala, ninguno de ellos es suficiente para encontrar una sólida explicación de este caso.

Hecha esta breve discusión teórica pasaremos a revisar el desarrollo de la tensión hídrica boliviana-chilena de los últimos setentaicinco años.

\section{Algo de historia sobre la tensión hidropolítica boliviana-chilena}

El conflicto boliviano-chileno alrededor de sus recursos hídricos compartidos, según la literatura académica sobre la dicotomía conflicto/cooperación, está referido a las tensiones no resueltas entre ambos países sobre la asignación, gestión y calidad de los recursos hídricos existentes en varios cursos de agua internacionales de escaso caudal existentes en su frontera. Los estudios bolivianos y chilenos han identificado varios problemas pendientes referidos a esos cursos de agua (Vergara Blanco, 2018). Se debe

\footnotetext{
6 Entre las más completas podemos mencionar 1) la «Base de datos internacional de eventos relacionados con el agua» de la Universidad de Oregon, Estados Unidos en: (https://transboundarywaters.science.oregonstate.edu/ content/international-water-event-database); 2) el "Atlas de riesgo hídrico» elaborado por Instituto de recursos Mundiales (WRI por sus siglas en inglés) en: https://www.wri.org/aqueduct; 3) el Programa Mundial de Evaluación de los Recursos Hídricos de la UNESCO (TWAP por sus siglas en inglés) en: http://www.unesco.org/new/en/ natural-sciences/environment/water/wwap/; y, 4) el Proyecto de los Correlatos de Guerra (COWP por sus siglas en inglés) en: https://correlatesofwar.org/

Para el caso de acuíferos como el Silala se puede revisar la información del «Centro Internacional de Evaluación de Recursos de Aguas Subterráneas» (IGRAC por sus siglas en inglés) en: https://www.un-igrac.org/.

En lo referido a los conflictos históricos por el agua se puede consultar «La cronología de los conflictos por el agua» en: http://www.worldwater.org/water-conflict/
} 
precisar que la gran mayoría de esos estudios se ha concentrado en dos temas: 1) la captación de una fracción de las aguas del río Lauca para llevarlas hacia la vertiente del Pacífico y 2) el uso de las aguas del Silala.

Sin embargo, se ha podido verificar que muy pocos de esos estudios han explorado a profundidad una característica importante en la generación de la tensión e interacción hidropolítica boliviano-chilena en torno a sus cursos de agua internacionales. Esta característica se vincula a los «problemas subyacentes» identificados por Newton (2007). Específicamente nos referimos a la explícita vinculación con el problema político bilateral más importante que existe entre ambos países: el acceso soberano de Bolivia al océano Pacífico. Dicha vinculación se hizo más evidente a partir de la cuarta década del Siglo pasado (Correa Vera, 31 de octubre de 2020; Correa Vera y García Pinzón, 2013; De Cossío Klüve, 2011).

Durante las negociaciones marítimas boliviano-chilenas - que tuvieron lugar en Santiago de Chile entre 1946-1952 - se planteó, por primera vez en la historia bilateral de ambos países, un intercambio de "tierra por agua.» Esta propuesta chilena significaba, en la práctica, que Bolivia compensaría a Chile con los recursos hídricos de lago Titicaca y del río Desaguadero ${ }^{7}$ a cambio de la cesión chilena a Bolivia de una franja de territorio de diez kilómetros de ancho que conectaría nuevamente a Bolivia con el mar, en donde se incluirían el ferrocarril La Paz-Arica y se construiría un puerto boliviano en la región de Arica (De Cossío Klüve, 2011). De haberse concretado esta propuesta inicial se hubiera producido una negociación de fondo sobre la mediterraneidad de Bolivia asociada a sus cursos de agua internacionales.

Se conoce que el tema del río Lauca, sobre el cual discutían ambos países desde al año 1939, fue tratado simultáneamente con las mencionadas negociaciones marítimas. Sin embargo, hasta el momento no se ha encontrado evidencia de su vinculación con el paquete de compensaciones hídricas solicitadas por Chile para que Bolivia accediese al océano Pacífico a través de la mencionada franja de diez kilómetros de ancho (De Cossío Klüve, 2011; De Iturralde Chinel, 1963; Ministerio de Relaciones Exteriores de Chile, 1963; Ministerio de Relaciones Exteriores y Culto del Estado Plurinacional de Bolivia, 1962).

Al fracasar este intento de negociaciones marítimas boliviano-chilenas de manera definitiva en el año 1952, el tema del uso chileno de los recursos hídricos bolivianos no se volvió a tratar en un contexto marítimo hasta una década después.

\footnotetext{
7 Que son dos cursos de agua internacionales exclusivamente peruano-bolivianos y sujetos a un régimen jurídico del cual Chile no es parte.
} 
La compensación boliviana con recursos hídricos a cambio de una salida soberana al océano Pacífico fue expresada con claridad por autoridades bolivianas, a principios de la década de 1960, con la frase "agua dulce por agua salada». De esa manera se graficó la conexión entre ambos temas (Fellmann Velarde, 1967). En este momento Bolivia y Chile ya no pensaban utilizar los recursos hídricos del altiplano peruanoboliviano, en especial los del lago Titicaca. En esta época la tensión hidropolítica entre ambos se centró alrededor del río Lauca (De Iturralde Chinel, 1963).

Este curso de agua internacional tiene 225 kilómetros de largo, de los cuales 75 se ubican en Chile y los restantes 150 en Bolivia (Vergara Blanco, 2018; Urquidi Barrau, 2005; Glassner, 1970). Nace en lagunas y bofedales (ciénagas de Parinacota, y las lagunas de Cotacotani y Chungará) situados en territorio chileno a más de 4400 metros de altura recorriéndolo hasta que ingresa a territorio boliviano para finalmente desembocar en el salar de Coipasa que es uno de los cuerpos de agua principales que integran el sistema TDPS (Autoridad Binacional Autónoma de la Cuenca del Sistema [ALT], s.f.).

Su caudal es modesto y variable ya que fluctúa entre los 100 a $560 \mathrm{l} / \mathrm{s}$ según la época estival o invernal ${ }^{8}$. Este curso de agua internacional es utilizado por Chile para generar energía hidroeléctrica y regar cultivos en el valle de Azapa en Arica (Bazoberry Quiroga, 2014).

En el mes de junio de 1939 el presidente de Chile, Pedro Aguirre Cerda, anunció en Arica su intención de captar parte de los recursos hídricos del Lauca para conducirlos, mediante un canal y un túnel, hacia el valle ariqueño de Azapa con fines de irrigación y, más tarde, para generar electricidad. En julio de ese mismo año Bolivia expresó, ante la Embajada de Chile en La Paz, sus reservas al proyecto hidráulico chileno. Bolivia expresó en que esa transferencia de agua de una cuenca a otra le causaba graves perjuicios. Chile, por el contrario, replicó en que tenía derecho a utilizar un porcentaje de los recursos hídricos de este curso de agua internacional, en conformidad con los principios aplicables del derecho internacional consuetudinario sobre la materia. Bolivia respondió que, de acuerdo con esos mismos principios, Chile debería obtener su consentimiento debido a que el «desvío» del río Lauca modificaría las condiciones hidrológicas y el régimen natural de este curso de agua internacional.

\footnotetext{
8 Si se compara el caudal del Lauca con el del río Rímac, por ejemplo, que en sus 204 kilómetros de largo acumula un caudal promedio de 25800 litros por segundo (1/s) nos percatamos del escaso caudal del Lauca. Al efectuar la misma comparación con el río Maipo verificamos que este, en sus 250 kilómetros de largo, tiene un caudal promedio de 92300 l/s, cifra muy superior a la del Lauca. Si comparamos el caudal del Lauca con los del Nilo (2 830000 l/s) o del Tigris (1 500000 l/s) la diferencia es abismal.
} 
La controversia se prolongó, con altibajos, por más de dos décadas y llegó a un punto álgido a principios de la década de 1960 .

En 1962, el presidente de Chile, Jorge Alessandri Rodríguez, decidió abrir las compuertas del canal de derivación para conducir parte de los recursos hídricos del río Lauca hacia el valle de Azapa y la ciudad de Arica. Como respuesta Bolivia rompió sus relaciones diplomáticas con Chile en abril de ese año (Ministerio de Relaciones Exteriores y Culto del Estado Plurinacional de Bolivia y DIREMAR, 2014), pidió a la OEA que condenara lo que calificó como una agresión chilena (Alcázar Chávez, 2017) y solicitó que se apliquen las disposiciones del TIAR (Tratado Interamericano de Asistencia Recíproca) en su contra (Ministerio de Relaciones Exteriores y Culto del Estado Plurinacional de Bolivia, 1962).

La OEA adoptó en el mes de mayo de ese año una resolución — por 18 votos a favor y ninguno en contra- en la que se pedía a ambos países resolver este asunto pacífcamente sin mencionar ningún tipo de agresión ni mucho menos el TIAR invocado por Bolivia. En setiembre de 1962 Bolivia anunció que suspendía su asistencia a todos los mecanismos de la OEA para retornar a ellos en octubre de ese mismo año. Como ya se mencionó, a raíz de esta controversia Bolivia rompió sus relaciones diplomáticas con Chile?.

La tensión hidropolítica entre ambos países por este curso de agua internacional sigue sin una solución definitiva. Este hecho fue recordado por el presidente Evo Morales cuando anunció el 23 de marzo de 2011, con ocasión de la conmemoración del Día del Mar, que el diferendo por el río Lauca, junto con el del Silala, podría ser llevado ante tribunales internacionales para ser resuelto.

El tema del uso de los cursos de agua internacionales boliviano-chilenos no se volvió a tratar bilateralmente en un contexto marítimo hasta que tuvieron lugar, entre 1975-1978, las negociaciones marítimas iniciadas con el abrazo de Charaña. En el marco de estas negociaciones, Chile le propuso a Bolivia «aprovechar la totalidad de las aguas del río Lauca» como una de las compensaciones exigidas para su acceso soberano al mar (Salazar Paredes et al., 2012; Prudencio Lizón, 2011; Ministerio de Relaciones Exteriores de Chile, 1979). Este planteamiento no llego a concretarse debido al fracaso definitivo de las negociaciones marítimas en 1979.

Ocho años después, en el marco de las negociaciones marítimas del «enfoque fresco» que tuvieron lugar en 1987, el canciller boliviano Guillermo Bedregal le entregó a

\footnotetext{
9 Debe recordarse que Bolivia reabrió sus relaciones diplomáticos con Chile por un período de tres años (19751978) para negociar con ese país una salida al océano Pacífico a través de una franja de 10 kilómetros de ancho ubicada al norte de Arica. Al no conseguir su objetivo marítimo Bolivia volvió a romper sus relaciones diplomáticas con Chile. Esta situación se mantiene hasta la fecha.
} 
su homólogo chileno, el canciller Jaime del Valle, una carta en la que, entre otros conceptos, le reiteraba «[...] la posición boliviana para resolver en forma diplomática la cuestión de la desviación del río Lauca, que para la Cancillería boliviana era un caso pendiente de solución mientras que para Chile era un caso resuelto» (Ministerio de Relaciones Exteriores y Culto del Estado Plurinacional Bolivia, 1988).

Sobre esta base los equipos de negociadores boliviano-chilenos reunidos en el mes de abril de 1987 en la ciudad de Montevideo (Uruguay) conversaron, junto con muchos otros temas, sobre sus cursos de agua internacionales. La delegación boliviana propuso a la de Chile la creación de una Comisión Mixta orientada a estudiar el aprovechamiento racional de los recursos hídricos comunes, es decir, los del río Lauca y otros pequeños cursos de agua binacionales. La delegación chilena solicitó información sobre las fuentes y cantidad de recursos hídricos permanentes que se aprovecharían y si estos eran de libre disposición de Bolivia o si se requería el acuerdo del Perú. A este requerimiento chileno la delegación boliviana respondió que los cursos de agua internacionales que se estaba negociando eran patrimonio exclusivo de Bolivia y no se requería el acuerdo del Perú ${ }^{10}$. Las negociaciones marítimas del «enfoque fresco» no prosperaron debido a la negativa chilena para seguir avanzando.

\section{El Silala aparece en el horizonte marítimo}

Diecinueve años después, en 2006, el tema de los recursos hídricos bolivianochilenos se incluyó en la Agenda de los trece puntos como punto sétimo («Silala y recursos hídricos»). El objetivo esencial de esta agenda global fue negociar el acceso soberano de Bolivia al océano Pacífico junto con otros importantes temas atingentes a la relación entre Bolivia y Chile (Bruce St John, 2020). Para afinar los detalles de los posibles acuerdos hídricos a los que podrían arribar, ambos países decidieron mantener la operatividad del «Grupo de Trabajo Bolivia - Chile sobre el tema del Silala». Se debe tener en consideración que este Grupo de Trabajo se estableció en 2004 (Ministerio de Relaciones Exteriores y Culto del Estado Plurinacional Bolivia y Ministerio de Relaciones Exteriores Chile, 28 de julio de 2009), con la finalidad de buscar una solución a la tensión hidropolítica que emergió sobre el Silala desde 1996 (Bazoberry Quiroga, 2003).

Por su pequeño caudal, la escasa población que vive en su área de influencia y los usos actuales del agua se percibe rápidamente que el Silala no tiene las particularidades de tensión hidropolítica que caracterizan a ríos como el Nilo, el Colorado, el Indo u

\footnotetext{
10 Tal como hubiera ocurrido si Bolivia volvía a contemplar el ofrecimiento del agua del lago Titicaca como compensación para su salida al mar como en 1950.
} 
otros grandes ríos y acuíferos del planeta. Tampoco es probable que se desencadene una guerra por la disputa sobre este curso de agua. Hasta la fecha el conflicto ha sido más discursivo, embebido en la estrategia boliviana del regreso al mar y recientemente elevado a la consideración de la Corte Internacional de Justicia.

El agua del Silala surge de un acuífero confinado ubicado en una ecorregión de clima árido muy similar a la que existe en algunas zonas del relieve andino peruano, en particular, las del sur del país (Peñaherrera del Águila, 1987; Brack Egg, 1987; Ponce de León Bardalez, 1994; Rundel Villagra et al., 2007; Young, León, Jørgensen y Ulloa Ulloa, 2007; Verbist et al., 2010). Esta ecorregión, llamada puna, se ubica por encima de los 3800 m.s.n.m. y se caracteriza por tener temperaturas bajas (entre $20^{\circ}$ y -100), escasas precipitaciones ( $4 \mathrm{~mm}$ en promedio anual), ríos y riachuelos de poco caudal, así como flora y fauna variada pero no muy abundante (Brack Egg, 1987).

El acuífero del Silala se ubica entre los $22^{\circ} 8^{\prime}$ de latitud sur y los $68^{\circ} 30^{\prime}$ de longitud oeste en el cantón Quetena de la provincia de Sur Lípez en el departamento boliviano de Potosí (Bazoberry Quiroga, 2003), colindando con la región Antofagasta de Chile11 muy cerca al complejo minero de Chuquicamata.

El afloramiento de agua del Silala en territorio boliviano abarca una superficie de once hectáreas y comprende alrededor de sesenta ojos de agua (Bazoberry Quiroga, 2014). Su fuente es un acuífero originado hace más de diez mil años por depósitos de agua fluvioglaciares (Bazoberry Quiroga, 2003). En 1908 obreros de la compañía de Salitres y Ferrocarril de Antofagasta empezaron a abrir zanjas y pozos para conectar todos los ojos de agua y canalizarlos hacia Chile (Fernández Ortiz, 2007, pp. 226-227). El canal colector principal construido para este efecto fue rudimentario. En la actualidad el canal principal tiene 120 centímetros de ancho y 150 centímetros de profundidad y conduce alrededor de 250 litros de agua por segundo hacia Chile (Bazoberry Quiroga, 2003).

Como ya se señaló, Bolivia y Chile iniciaron negociaciones en el año 2006 sobre sus recursos hídricos transfronterizos asociándolos a la solución del problema mediterráneo de Bolivia. El instrumento de esta nueva negociación marítima fue el Mecanismo de Consultas Políticas Bolivia-Chile. Esta instancia bilateral consignó en

\footnotetext{
11 Se tiene conocimiento que, en territorio chileno, a 1200 metros de la frontera con Bolivia, existe un afloramiento natural de agua en la zona denominada Quebrada Negra. El acuífero que alimenta los manantiales de esta quebrada podría estar relacionado con el que alimenta al Silala dada su cercanía al territorio boliviano. Aunque se conoce muy poco sobre las características hidrogeológicas del acuífero que alimentaría ambos afloramientos, se podría suponer que se trata de un único acuífero transfronterizo confinado de aguas fósiles que es compartido por Bolivia y Chile. De comprobarse la interconexión entre los dos afloramientos naturales de agua subterránea se trataría de un curso de agua internacional independientemente si el agua que surge en el territorio boliviano fluye superficialmente o no hacia el territorio chileno.
} 
el Acta de su XX Reunión, realizada en la ciudad de La Paz el 30 de junio de 2009, los acuerdos a los que arribó el «Grupo de Trabajo Bolivia-Chile sobre el tema del Silala». En dicha Acta se registró que el Grupo de Trabajo sobre el Silala presentó su informe final sobre el proyecto de Acuerdo Inicial sobre este «sistema hídrico». También recomendó la adopción de ese acuerdo «en fecha próxima» (Ministerio de Relaciones Exteriores Chile, 2020a).

El referido proyecto de Acuerdo Inicial12 fue rubricado por los viceministros de Relaciones Exteriores de Bolivia y de Chile el 13 de noviembre de 2009 (Ministerio de Relaciones Exteriores de Chile, 2010, p. 44; Fundación Milenio, 2010). Su texto fue ampliamente difundido en la prensa de ambos países. En él se estableció que serviría como una base para alcanzar un nuevo acuerdo de largo plazo que consideraría los aprovechamientos hídricos existentes y el uso sustentable de las aguas del Silala (Ministerio de Relaciones Exteriores y Culto del Estado Plurinacional de Bolivia y Ministerio de Relaciones Exteriores Chile, 28 de julio de 2009).

Para efectos de este estudio debe destacarse que el objeto de ese preacuerdo fue la preservación, sostenibilidad, uso y aprovechamiento del «sistema hídrico» del Silala o Siloli para beneficio de ambos países (Ministerio de Relaciones Exteriores y Culto del Estado Plurinacional de Bolivia y Ministerio de Relaciones Exteriores Chile, 28 de julio de 2009, artículo primero).

El preacuerdo establecía, entre sus disposiciones más importantes, que solo se aplicaría al volumen de agua de dicho sistema "que fluye superficialmente a través de la frontera desde el Estado Plurinacional de Bolivia hacia la República de Chile», del cual el 50 por ciento le correspondería a Bolivia (Ministerio de Relaciones Exteriores y Culto del Estado Plurinacional de Bolivia y Ministerio de Relaciones Exteriores Chile, 28 de julio de 2009, artículo segundo). También establecía que sobre la base de futuros estudios técnicos y científicos, se repartiría la otra mitad entre ambos países (Ministerio de Relaciones Exteriores y Culto del Estado Plurinacional de Bolivia y Ministerio de Relaciones Exteriores Chile, 28 de julio de 2009, artículos segundo y sexto). Se aseguraba a Bolivia la libre disponibilidad y dominio sobre el agua superficial del Silala que le correspondiera para los usos que estimara convenientes como su uso interno o su venta a Chile (Ministerio de Relaciones Exteriores y Culto del Estado Plurinacional de Bolivia y Ministerio de Relaciones Exteriores Chile, 28 de julio de 2009, artículo tercero). Ambas Partes se comprometían a mantener el caudal y calidad del agua que "fluye a través de la frontera» y a evitar que futuras obras afecten ese caudal y su calidad (Ministerio de Relaciones Exteriores y Culto del Estado Plurinacional de Bolivia y Ministerio de Relaciones Exteriores Chile, 28 de julio de 2009, artículo cuarto).

12 Para más información véase: Bazoberry Quiroga, 2014, pp. 184-190; Correa Vera y García Pinzón, 2013, p. 106. 
Al conocerse en Bolivia el alcance del preacuerdo al que se había llegado con Chile la población boliviana reaccionó airadamente. Un tema que fue medular en las críticas formuladas al gobierno del presidente Morales fue la existencia de una «deuda histórica» en la que habría incurrido Chile por haber utilizado por casi cien años los recursos hídricos del Silala sin compensar debidamente a Bolivia. Esta deuda no estaba contemplada en el texto del preacuerdo.

En particular, la oposición a este proyecto de acuerdo con Chile por parte de la población de Potosí (donde se ubica el acuífero del Silala) fue importante para evitar que se formalizara. Esta población, organizada alrededor del "Comité Cívico Potosinista» (COMCIPO), sostuvo invariablemente que si en el preacuerdo con Chile sobre el Silala no se incluía la «deuda histórica» se opondrían a su aprobación.

Hacia el mes de diciembre de 2010, momento en el que las conversaciones marítimas boliviano-chilenas llegaban a un punto muerto, los delegados boliviano-chilenos ante el Mecanismo de Consultas Políticas Bolivia-Chile expresaban que:

Ambas delegaciones conversaron respecto al proceso de socialización en Bolivia del Acuerdo Inicial del Silala; planteando Bolivia el tema de la deuda histórica. En virtud a que no existen coincidencias al respecto, ambas delegaciones acordaron que el Grupo de Trabajo sobre la temática del Silala se reúna nuevamente con el objetivo de conocer, analizar y responder, todas las propuestas surgidas a raíz del proceso de socialización del Acuerdo Inicial. Dicho Grupo de Trabajo deberá elevar un informe a la próxima reunión del Mecanismo de Consultas Políticas. (Ministerio de Relaciones Exteriores y Culto del Estado Plurinacional de Bolivia y Ministerio de Relaciones Exteriores Chile, 2010, p. 9)

El Grupo de Trabajo sobre el Silala no llegó a convocarse y las reuniones del Mecanismo de Consultas Políticas se encuentran suspendidas. De esta manera concluyeron las negociaciones boliviano-chilenas sobre el Silala y otros recursos hídricos, sin registrarse a la fecha ninguna aproximación bilateral sobre este tema.

\section{La controversia por las aguas del Silala se presenta ante la Corte Internacional de Justicia $(\mathrm{CIJ})$}

A partir del año 2011 — detenidas definitivamente las negociaciones marítimas organizadas en torno a la «Agenda de los trece puntos»— el gobierno boliviano inició una campaña mediática para sensibilizar a su población sobre una eventual demanda en contra de Chile ante tribunales internacionales por lo que calificaba como el uso unilateral de sus recursos hídricos transfronterizos. En esa campaña ocuparon un lugar destacado el Lauca y el Silala. En un discurso pronunciado el 23 de marzo de 2016 por el presidente Evo Morales, con ocasión de la celebración del Día del Mar, dijo lo siguiente: 
Sin embargo, hermanas y hermanos, para que este propósito se haga realidad, resulta imprescindible que resolvamos los demás temas pendientes de la agenda bilateral, como el relativo al uso y aprovechamiento unilateral e ilícito por parte de Chile de las aguas bolivianas de los manantiales del Silala. Cada día, Chile se aprovecha ilegal y arteramente de ese recurso natural sin compensar ni un centavo. Este acto abusivo y arbitrario, que vulnera nuestro patrimonio, no puede continuar. [...] Hermanas y hermanos, por esta razón he instruido a Diremar (Dirección Estratégica de Reivindicación Maritima) que estudie las alternativas jurídicas para asumir la defensa de nuestras aguas del Silala, ante las instancias internacionales competentes. (Viceministerio de Comunicación del Estado Plurinacional de Bolivia, 23 de marzo de 2016)

En otro discurso pronunciado en Cochabamba, el 27 de marzo de 2016, el presidente Evo Morales anunció que Bolivia iba a "presentar la demanda ante La Haya, para sentar soberanía sobre las aguas del Silala desde el departamento de Potosí» (Viceministerio de Comunicación del Estado Plurinacional de Bolivia, 27 de marzo de 2016).

Simultáneamente a estas denuncias públicas, respondidas oportunamente por el gobierno chileno, Bolivia empezó a ejecutar varios proyectos productivos (criadero de truchas, planta embotelladora, irrigaciones y uso doméstico) para utilizar el agua del Silala y «sentar soberanía» sobre sus recursos naturales (DIREMAR, 28 de marzo de 2013). Chile planteó oficialmente sus reservas a estos proyectos.

El presidente Morales reiteró en abril de 2016 que demandaría a Chile ante instancias jurídicas internacionales. Esta afirmación se produjo en la ceremonia en que posesionó al exdiputado René Martínez como secretario general del Consejo de Defensa del Silala y otros recursos hídricos en la frontera con Chile (Viceministerio de Comunicación del Estado Plurinacional de Bolivia, 30 de marzo de 2016; Viceministerio de Comunicación del Estado Plurinacional de Bolivia, 16 de mayo de 2016; Viceministerio de Comunicación del Estado Plurinacional de Bolivia, 17 de mayo de 2016).

Cuando todos los indicios y señales hacían prever que sería Bolivia quien llevaría a Chile nuevamente ante la Corte Internacional de Justicia (CIJ), ${ }^{13}$ en un giro jurídico

\footnotetext{
13 Se debe recordar que el 24 de abril de 2013 Bolivia inició un proceso ante la CIJ contra Chile en relación con una controversia sobre «la obligación de Chile de negociar de buena fe y efectivamente con Bolivia para llegar a un acuerdo que otorgue a Bolivia un acceso soberano al océano Pacífico». La Corte emitió su sentencia de fondo el 1 de octubre de 2018. En ese documento la Corte concluyó que ninguna de las bases propuestas establecía una obligación para Chile de negociar el acceso soberano de Bolivia al océano Pacífico. Agregó que su conclusión no debe entenderse «en el sentido de que se impide a las Partes continuar su diálogo e intercambios, en un espíritu de buena vecindad, para abordar los problemas relacionados con la situación sin litoral de Bolivia, la solución a la que ambas habían reconocido como un asunto de interés mutuo. Con la voluntad de las Partes, se podrían emprender negociaciones significativas».
} 
y político inesperado, Chile interpuso ante la CIJ una demanda en contra de Bolivia para determinar el estatuto jurídico del uso de las aguas del Silala.

En la solicitud de apertura de este procedimiento, presentada el 6 de junio de 2016, Chile pidió a la Corte que se pronuncie y declare que: «El sistema del río Silala, junto con las partes subterráneas de su sistema, es un curso de agua internacional, cuyo uso se rige por el derecho internacional consuetudinario» (International Court of Justice [ICJ], 6 de junio de 2016).

La posición de Chile, expresada por su Ministerio de Relaciones Exteriores, sostiene que el Silala pertenece a la hoya hidrográfica del río Loa y es afluente del río Inacaliri, que tributa al río San Pedro y, a través del río Loa, llega al mar (Ministerio de Relaciones Exteriores Chile, 2020b). En el año 2019, el canciller de Chile, Roberto Ampuero, sostuvo que:

Chile tiene toda la razón al plantear su posición y Bolivia no solamente va a tener que tratar de desmentir a Chile, sino que también va a tener que tratar de desmentir la ley de gravedad a la ciencia. (Ministerio de Relaciones Exteriores Chile, 28 de enero de 2019)

[...] es indudable que el río Silala es un río Internacional, que fluye desde las alturas de Bolivia de forma natural hacia Chile, por lo tanto, este es un asunto que tiene que ver con la ley de gravedad. Bolivia no puede desmentir la ley de gravedad, ha tratado de desmentir la posición de Chile, pero la ley de gravedad es muy clara, el agua fluye de lo alto hacia lo bajo, por lo que estamos muy tranquilos. (Ministerio de Relaciones Exteriores Chile, 24 de mayo de 2019)

El río Silala es un río internacional que cruza la frontera hacia nuestro país en forma natural. Para este efecto, hemos acompañado ante la Corte Internacional de Justicia mucha y abundante evidencia científica y jurídica que fortalece nuestra causa. (Ministerio de Relaciones Exteriores Chile, 24 de mayo de 2019)

La posición chilena se opone diametralmente a la de Bolivia. De acuerdo con la tesis de Bolivia las aguas del Silala están localizadas exclusivamente en su territorio, en el departamento de Potosí, cerca de la frontera con Chile. Estas aguas constituyen el afloramiento natural (bofedales) de las aguas de un acuífero confinado ubicado únicamente en territorio boliviano. Asimismo, Bolivia sostiene que estos recursos hídricos han sido canalizados sin su consentimiento hacia el territorio chileno desde 1908. En otras palabras, Bolivia plantea que el Silala no es un curso de agua internacional, sea como río o como un acuífero, y que Chile ha desviado inconsultamente sus aguas a través de canales construidos a principios del siglo XX (Bazoberry, 2003, pp. 89-91).

Se debe destacar sin embargo, que en los primeros meses de 2020 la administración interina boliviana anunció, a través de un comunicado de prensa de su Ministerio de Relaciones Exteriores, que durante el mandato del presidente Morales se había 
admitido ante la Corte Internacional de Justicia «[...] que una parte de las aguas del Silala fluyen de manera natural hacia Chile» (Ministerio de Relaciones Exteriores y Culto del Estado Plurinacional de Bolivia, 13 de febrero de 2020). De ser cierta esta afirmación, se habría modificado sustantivamente la posición boliviana para acercarse en algo a la de Chile.

$\mathrm{Al}$ momento de finalizar la redacción de este artículo, ambas partes ya han concluido con la presentación de sus alegatos escritos ante la CIJ. Se espera que la Corte determine próximamente la fecha en que se iniciará la fase oral del proceso. Sobre el particular la canciller de Bolivia, Karen Longaric expresó durante una conferencia de prensa en la ciudad de La Paz que esperaba que los alegatos orales del caso se produjesen entre los meses de abril o mayo de 2020 (Ministerio de Relaciones Exteriores y Culto del Estado Plurinacional de Bolivia y DIREMAR Bolivia, 20 de enero de 2020). Debido al impacto de la pandemia de la COVID-19 esta decisión de la Corte no se produjo como se preveía, por lo que se espera que la fase oral del proceso se inicie el 2021.

La conclusión de este caso contribuirá positivamente al desarrollo del derecho de los cursos de agua internacionales si la Corte se enfoca en la parte superficial del Silala, pero también puede esclarecer el derecho de los acuíferos transfronterizos en caso de que decida encarar también las partes subterráneas del sistema hidrográfico del Silala (De Cossío Klüver, 2019).

Las posibilidades para que la cooperación sobre el Silala prevalezca sobre el conflicto, una vez que se conozca el fallo de la CIJ, dependerán de la voluntad política con la que ambos países decidan gestionar el uso de esos recursos hídricos y si se vuelve a vincular o no al tema del acceso de Bolivia al océano Pacífico.

\section{Conclusiones}

Los estudios realizados para determinar cuáles son los factores que exacerban las tensiones hídricas en contextos transfronterizos han propuesto diversos indicadores para identificarlos. Pocos de esos estudios se han aplicado al caso del Silala o, en general, a los cursos de agua internacionales ubicados en las zonas áridas y semiáridas de América del Sur.

Hace más de cien años que en el Silala no se ha construido una infraestructura importante para extraer un volumen mayor de agua del sistema. Tampoco se conoce la existencia de nuevos proyectos de infraestructura hidráulica. De acuerdo con las metodologías discutidas en este texto no deberían existir tensiones hidropolíticas entre Bolivia y Chile por el uso de las aguas del Silala debido a que no existen nuevos proyectos de derivación de su agua hacia Chile. 
Es un hecho que entre Bolivia y Chile existe un alto nivel de tensión hidropolítica que tiene su origen en factores no contemplados por los estudios revisados en este texto. En el caso del Silala se puede observar que, mientras no se resuelva el problema político mayor del acceso soberano de Bolivia al océano Pacífico, los conflictos boliviano-chilenos por esos recursos hídricos podrían seguir teniendo un lugar preeminente en la agenda bilateral entre ambos países.

También se puede afirmar que los factores que influyen de manera importante en la generación de la tensión hidropolítica existente entre Bolivia y Chile en torno a los recursos hídricos del Silala se encuentran más allá de los factores que usualmente constituyen la gestión y gobernanza del agua (cantidad, calidad, infraestructura existente y en proyecto, gestión, marco legal, participación de las partes interesadas, etc.). El Silala es un caso en el que la tensión hidropolítica no se ha resuelto cooperativamente debido a la existencia de factores políticos profundos vinculados a los «problemas subyacentes» identificados por Newton.

Del breve recuento histórico sobre la tensión hidropolítica boliviano-chilena se puede comprobar que ambos países han vinculado, en más de una oportunidad y a lo largo de más de siete décadas, el uso y gestión de sus recursos hídricos transfronterizos, sobre todo los del Lauca y del Silala, a la eventual resolución del problema mediterráneo de Bolivia.

Un factor exacerbante de la tensión hídrica entre ambos países es que, a pesar de que en el año 2010 se inicializó un proyecto de acuerdo sobre el Silala/Siloli, actualmente no existe un marco institucional ni legal para regular el uso secular e ininterrumpido que hace Chile del agua del Silala. También se debe tener en consideración que la situación existente no ha derivado en un conflicto armado sino en un alto nivel de tensión bilateral y desconfianza mutua.

En la eventualidad que el Fallo de la CIJ sobre el Silala determine que es un curso de agua internacional (sea por su flujo superficial, por su flujo subterráneo o por ambos) quedará abierta la posibilidad de que Bolivia y Chile puedan llegar a negociar en el futuro un Acuerdo para la gestión conjunta de los recursos hídricos del Silala y, como parte de él, se pueda establecer algún tipo de organización de cuenca a través de la cual se gestione el recurso. Cabe recordar que ese fue el sentido del preacuerdo del año 2010.

En resumen, estamos en presencia de un caso en el que el conflicto es gatillado por factores políticos y económicos que trascienden la gobernanza del agua, pero en el que se podría alcanzar un acuerdo cooperativo cuando existan las condiciones apropiadas para concluirlo. 


\section{Referencias bibliográficas}

Alcázar Chávez, L.F. (2017). 250 Agresiones de Chile a Bolivia por la cuestión maritima. Cámara de Senadores Asamblea Legislativa Plurinacional de Bolivia. https://web. senado.gob.bo/sites/default/files/publicaciones/250\%20AGRESIONES\%20DE\%20 CHILE\%20ARCHIVO\%20FINAL\%20peque\%20\%281\%29.pdf.

Autoridad Binacional Autónoma de la Cuenca del Sistema [ALT]. (s.f.). T.D.P.S. Plan director global binacional Perú-Bolivia : Cuenca : Titicaca-Desaguadero-Poopo-Salar. https:// portals.iucn.org/library/node/21721.

Bazoberry Quiroga, A. (2003). El Mito del Silala. Plural Editores.

Bazoberry Quiroga, A. (2014). Recursos hidráulicos de Bolivia. Plural Editores.

Brack Egg, A. (1987). Ecología de un país complejo. En Gran Geografía del Perú (vol. 2, pp. 175-319). 2da. ed. Editorial Juan Mejía Baca / Coedición Manfer.

Bruce St John, R. (2020). Bolivia: Geopolitics of a Landlocked State. Europa Country Perspectives. Routledge, Taylor \& Francis Group.

Corporación Nacional del Cobre de Chile [CODELCO]. (2019). Memoria Anual 2019. https://www.codelco.com/memoria2019/

Correa Vera, L. (31 de octubre de 2020). Aguas dulces entre Chile y Bolivia: el Silala en su laberinto. Relaciones Internacionales, (45), 163-183. https://doi.org/10.15366/ relacionesinternacionales2020.45.007.

Correa Vera, L. (2019). Escenarios de Chile con Bolivia: 2019-2021. Academia Nacional de Estudios Políticos y Estratégicos (ANEPE).

Correa Vera, L. y García Pinzón, V. (2013). Turbulencias desde el mar: Chile y Bolivia. Si Somos Americanos. Revista de Estudios Transfronterizos, 3(1), 93-121.

Correa Vera, L. y Salas Maturana, A. (2015). Gobernabilidad, desarrollo y seguridad en las zonas extremas de Chile. Colección Investigaciones ANEPE 37. Academia Nacional de Estudios Políticos y Estratégicos (ANEPE).

De Cossío Klüver, M. A. (2011). Tierra por agua: Las Negociaciones Marítimas bolivianochilenas (1946-1952). [Tesis de maestría, Universidad Católica Boliviana San Pablo].

De Cossío Klüver, M. A. (2019). Un recurso natural invisible: los acuíferos transfronterizos. Agenda Internacional, 26(37), 137-157.

De Iturralde Chinel, L. (1963). La desviación del río Lauca por Chile. Empresa Industrial Grafica E. Burillo.

De Stefano, L., Petersen-Perlman, J. D., Sproles, E. A., Eynard, J. y Wolf, A. T. (2017). Assessment of Transboundary River Basins for Potential Hydro-Political Tensions. Global Environmental Change, (45), 35-46.

Dirección Estratégica de Reivindicación Marítima [DIREMAR]. (28 de marzo de 2013). Silala y Recursos Hídricos Internacionales. «Morales inaugura criadero de truchas en Silala y llama a sentar soberanía sobre recursos naturales». http://www.diremar.gob. bo/diremar/index.php/2013/03/28/morales-inaugura-criadero-de-truchas-en-silalay-llama-a-sentar-soberania-sobre-recursos-naturales/ 
Dolatyar, M. (1 de agosto de 2002). Hydropolitics: challenging the water-war thesis. Conflict, Security\&Development2(2),115-124.https://doi.org/10.1080/14678800200590612.

Elhance, Arun P. (1999). Hydropolitics in the Third World: Conflict and Cooperation in International River Basins. United States Institute of Peace Press.

Farinosi, F., Giupponi, C., Reynaud, A., Ceccherini, G., Carmona-Moreno, C., De Roo, A., Gonzalez-Sanchez, D. y Bidoglio, G. (2018). An Innovative Approach to the Assessment of Hydro-Political Risk: A Spatially Explicit, Data Driven Indicator of Hydro-Political Issues. Global Environmental Change, (52), 286-313. https://doi. org/10.1016/j.gloenvcha.2018.07.001.

Faundes Sánchez, C. (2008). El agua como factor estratégico en la relación entre Chile y los paises vecinos. Colección Investigaciones ANEPE 20. Academia Nacional de Estudios Políticos y Estratégicos (ANEPE).

Fellmann Velarde, J. (1967). Memorándum sobre Política Exterior Boliviana. (2a ed.). Librería Editorial Juventud.

Fernández Ortiz, R. (2007). Algunas consideraciones históricas, técnicas y políticas sobre el problema de las aguas del Silala: La posición boliviana. En Mar de fondo. Chile y Bolivia, un siglo de desencuentros (pp. 223-259). Santiago de Chile: Instituto de Estudios Avanzados de la Universidad Santiago de Chile.

Fundación Milenio. Compensación es venta: la historia del Silala. Informe Nacional de Coyuntura, 57. La Paz: Fundación Milenio.

Gallopín, G. C. (2012). Five Stylized Scenarios. UNESCO. Global Water Futures 20501. https://unesdoc.unesco.org/ark:/48223/pf0000215380

Ganter, C. (2015). Water Crises Are a Top Global Risk. World Economic Forum, 2015. https:// www.weforum.org/agenda/2015/01/why-world-water-crises-are-a-top-global-risk/

Glassner, M. I. (1970). The Rio Lauca: Dispute over an International River. American Geographical Society, 60(2), 192-207.

Gleick, P. Water Conflict Chronology. Water Conflict Chronology. Recuperado el 16 de agosto de 2020 de http://www.worldwater.org/conflict/map/

Gleick, P. e Iceland, C. (2018). Water, Security and Conflict. Issue Brief. WRI / Pacific Institute. Recuperado de https:/www.wri.org/publication/water-security-and-conflict.

Gleick, P., Iceland, C. y Trivedi, A. (2020). Ending Conflicts over Water. Solutions to Water and Security Challenges. WRI / Pacific Institute / WPS.

Intelligence Community Assessment [ICA]. (2012). Global Water Security. Office of the Director of National Intelligence.

International Court of Justice [ICJ]. (6 de junio de 2016). Requête Introductive d'instance enregistrée au Greffe de la Cour le 6 juin 2016. Différend Concernat le Statut et L'utilisation des Eaux du Silala (Chili c. Bolivie). https://www.icj-cij.org/fr/ affaire/162/introduction-instance

Meissner, R. (2016). Hydropolitics, Interest Groups and Governance. The Case of the Proposed Eрира Dam. Springer international Publishing. 
Ministerio de Relaciones Exteriores y Culto del Estado Plurinacional de Bolivia. (1962). La desviación del río Lauca. Antecedentes y documentos. Imprenta Nacional.

Ministerio de Relaciones Exteriores y Culto del Estado Plurinacional de Bolivia. (1988). Tricolor. Historia y proyecciones de paz, desarrollo e integración del diferendo maritimo Boliviano-Chileno. Editorial «Los Amigos del Libro» Werner Guttentag T.

Ministerio de Relaciones Exteriores y Culto del Estado Plurinacional de Bolivia. (13 de febrero de 2020). Sobre la controversia del Silala. http://www.cancilleria.gob.bo/ webmre/comunicado/3817

Ministerio de Relaciones Exteriores y Culto del Estado Plurinacional de Bolivia y DIREMAR. (2014). El libro del mar. Dirección Estratégica de Reivindicación Marítima (DIREMAR). http://www.diremar.gob.bo/diremar/index.php/libro-del-mar/

Ministerio de Relaciones Exteriores y Culto del Estado Plurinacional de Bolivia y DIREMAR. (20 de enero de 2020). Longaric anuncia reuniones para encarar fase de alegatos orales ante la CIJ por caso Silala. http://www.diremar.gob.bo/diremar/index. $\mathrm{php} / 2019 / 12 / 23 /$ longaric-anuncia-reuniones-para-encarar-fase-de-alegatos-oralesante-la-cij-por-caso-silala/

Ministerio de Relaciones Exteriores y Culto del Estado Plurinacional de Bolivia y Ministerio de Relaciones Exteriores de la República de Chile. (28 de julio de 2009). Acuerdo inicial sobre el Silala o Siloli. https:/www.internationalwaterlaw.org/documents/ regionaldocs/Silala/SilalaAgreement2009_Spanish.pdf

Ministerio de Relaciones Exteriores y Culto del Estado Plurinacional de Bolivia y Ministerio de Relaciones Exteriores de Chile. (2010). Acta de la XXII Reunión del Mecanismo de Consultas Politicas Bolivia-Chile. https://minrel.gob.cl/minrel_old/site/ artic/20100715/asocfile/20100715165143/acta_final.pdf

Ministerio de Relaciones Exteriores de Chile. (1963). La cuestión del Rio Lauca. Instituto Geográfico Militar.

Ministerio de Relaciones Exteriores de Chile. (1979). Historia de las Negociaciones ChilenoBolivianas 1975-1978. Gobierno de Chile.

Ministerio de Relaciones Exteriores de Chile. (2010). Hitos de la Politica Exterior de Chile. Gobierno de Chile.

Ministerio de Relaciones Exteriores de Chile. (28 de enero de 2019). Canciller Ampuero: «Bolivia no solamente va a tener que tratar de desmentir a Chile». https:// chileantelahaya.gob.cl/canciller-roberto-ampuero-bolivia-no-solamente-va-atener-que-tratar-de-desmentir-a-chile-sino-que-tambien-va-a-tener-que-tratar-dedesmentir-la-ley-de-gravedad-2/

Ministerio de Relaciones Exteriores de Chile. (24 de mayo de 2019). Canciller: «Estamos muy tranquilos con la solidez de nuestros argumentos». Chile Ante La Haya. https://chileantelahaya.gob.cl/estamos-muy-tranquilos-con-la-solidez-de-nuestros-argumentos/.

Ministerio de Relaciones Exteriores de Chile. (2020a). Acta de la XX Reunión del Mecanismo de Consultas Politicas Bolivia-Chile. https://minrel.gob.cl/acta-de-la-xx-reunion-delmecanismo-de-consultas-politicas-bolivia/minrel/2009-06-30/214914.html. 
Ministerio de Relaciones Exteriores de Chile. (2020b). Río Silala. https://chileantelahaya. gob.cl/caso-silala/rio-silala/.

Newton, J. T. (2007). Hydropolitical Vulnerability of South America's International Water Resources. En Hydropolitical Vulnerability and Resilience along International Waters. Latin America and the Caribean. UNEP.

Otchet, A. (2001). Saber-Rattling Among Thirsty Nations. The UNESCO Courrier. http:// www.unesco.org/courier/2001_10/uk/doss01.htm

Peñaherrera del Águila, C. (1987). Geografía física del Perú. En Gran Geografía del Perú (2a ed., Vol. 1, pp. 1-222). Editorial Juan Mejía Baca / Coedición Manfer.

Ponce de León Bardalez, R.G. (1994). El Perú y sus recursos. Atlas geográfico y económico. Auge S. A. Editores.

Prudencio Lizón, R. (2011). Historia de la negociación de Charaña. Plural Editores.

Reed, D. (2017). Water, Security and U.S. Foreign Policy. Taylor and Francis Group.

Rundel, P. W., Villagra, P. E., Dillon, M. O., Roig-Juñent, S., y Debandi, G. (2007). Arid and Semi-Arid Ecosystems. En T. Veblin, K. Young y A. Orme. The Physical Geography of South America (pp. 158-183). Oxford University Press. DOI: 10.1093/ oso/9780195313413.003.0018

Salazar Paredes, F., Gumucio Granier, J., Orozco Padilla, F. y Salazar Machicado, L. (2012). Charaña. Una negociación boliviana 1975-1978. Ediciones CERID.

Torres Armas, W. G. (2000). Política exterior en materia de cursos de agua Internacionales. En Bolivia: temas de la agenda internacional (pp. 121-158). Ministerio de Relaciones Exteriores y Culto de Bolivia.

Turton, A. y Henwood, R. (2002). (Eds.), Hydropolitics in the Developing World: A Southern African Perspective. African Water Issues Research Unit of the Centre for International Political Studies, University of Pretoria.

UNEP - United Nations Environment Program. (2007). Hydropolitical Vulnerability and Resilience along International Waters. Latin America and the Caribean. UNEP.

Unesco. (27 de setiembre de 2018). PCCP. From Potential Conflict to Cooperartion Potential. Institutional. International Hydrological Programme. https://groundwaterportal.net/ project/pccp

UN-Water. (2013). Water Security and the Global Water Agenda. United Nations University. http://www.unwater.org/publications/water-security-global-water-agenda/

Urquidi Barrau, F. (2005). Recursos hídricos en la frontera boliviano-chilena (Silala-Lauca). En Política exterior en materia de recursos hídricos (pp. 37-70). UDAPEX - PNUD.

Verbist, K., Santibañez, F., Gabriels, D. y Soto, G. (2010). Atlas de zonas áridas de América Latina y el Caribe. Programa Hidrológico Internacional (PHI) de la Oficina Regional de Ciencia para América Latina y el Caribe de la Unesco y el Centro del Agua para Zonas Áridas de América Latina y el Caribe (CAZALAC). https://www.cazalac.org/ publico/index.php?id=19\&L=0.

Vergara Blanco, A. (2018). Aguas transfronterizas de Chile: Esquema de conflictos y acuerdos bilaterales. En PUCP. El derecho y la gestión de aguas transfronterizas. Quintas Jornadas 
de Derecho de Aguas (pp. 217-240). Fondo Editorial de la Pontificia Universidad Católica del Perú.

Viceministerio de Comunicación del Estado Plurinacional de Bolivia. (23 de marzo de 2016). Discurso del Presidente del Estado Plurinacional de Bolivia, Evo Morales Ayma, en el Día del Mar.

Viceministerio de Comunicación del Estado Plurinacional de Bolivia. (27 de marzo de 2016). El Gobierno de Bolivia demandará a Chile, en La Haya, por el respeto a las aguas del Silala. https://comunicacion.gob.bo/?q=20160405/20964

Viceministerio de Comunicación del Estado Plurinacional de Bolivia. (30 de marzo de 2016). Gobierno creará nuevo equipo internacional de expertos en temas hidricos para la demanda contra Chile por el Silala. https://comunicacion.gob.bo/?q=20160405/20967

Viceministerio de Comunicación del Estado Plurinacional de Bolivia. (16 de mayo de 2016). Presidente Evo Morales durante la posesión de Rene Martínez como secretario general del Consejo de Defensa del Silala y otros recursos hidricos en frontera con Chile. https:// comunicacion.gob.bo/?q=20160516/21257

Viceministerio de Comunicación del Estado Plurinacional de Bolivia. (17 de mayo de 2016). Evo Morales: La creación de la Dirección de Defensa del Silala se debe a que llegó la hora de sentar soberanía por los recursos naturales. https://comunicacion.gob. bo/?q=20160518/21278

Waterbury, J. (1979). Hydropolitics of the Nile Valley. Contemporary Issues in the Middle East. Syracuse University Press.

World Economic Forum [WEF]. (2018) The Global Risks Report 2018. 13th Edition. WEF. https://www.weforum.org/reports/the-global-risks-report-2018/

World Economic Forum [WEF]. (2020). The Global Risks Report 2020. (15 a ed.) https:// www.weforum.org/reports/the-global-risks-report-2020/

Wolf, A. T. (2007). Hydropolitical Vulnerability and Resilience. En Hydropolitical Vulnerability and Resilience along International Waters. Latin America and the Caribean (pp. 1-16). UNEP.

Wong, C. M., Williams, C. E. Pittock, J. y Schelle, P. (2007). World's Top 10 Rivers at Risk. WWF International. https://wwf.panda.org/?108620/Worlds-Top-10-Rivers-at-Risk

Young, K. R., León, B., Jørgensen, P. M. y Ulloa Ulloa, C. (2007). Tropical and Subtropical Landscapes of the Andes. En The Physical Geography of South America (Vol. 8, pp. 200-216). Oxford University Press.

Zambrano, P. (2015). Conflicto por el Silala, una oportunidad para la cooperación - ANEPE - Academia Nacional de Estudios Políticos y Estratégicos - Ministerio de Defensa Nacional. Sitio web Academia Nacional de Estudios Políticos y Estratégicos Ministerio de Defensa Nacional (blog).

Fecha de recepción: 8 de febrero de 2021 cha de aprobación: 3 de julio de 2021 